\title{
Networked Video Smoke and Fire Monitoring System Based on DM642 and i.MX27
}

\author{
Zhang Hong ${ }^{1}$, Feng Xianzhou ${ }^{1}$, Lyu Ming ${ }^{2 *}$, Zhang $\mathrm{Jie}^{3}$, Li Haiyan ${ }^{3}$ and Liu \\ $\mathrm{Lei}^{3}$ \\ ${ }^{1} 713$ Research Institue of China Shipbuilding Industry Corporation \\ ${ }^{2}$ Simulation Equipment Business Department, North Information Control \\ Institute Group Co., Ltd., Nanjing, China. \\ ${ }^{3}$ School of Automation, Nanjing University of Science and Technology \\ liuleisdly@163.com
}

\begin{abstract}
In this paper, a networked video smoke and fire detector based on TMS320DM642 and i.MX27 is designed. The design of the system hardware, software platform and detection algorithm will be introduced. In order to improve the accuracy of smoke detection and reduce the false alarm rate, color, energy and structural similarity metric algorithm is organically combined in the detection algorithm. Flame detection uses dual band of infrared and visible light. Through the double determination of infrared and visible light, compared with the general image processing based flame recognition, the accuracy and stability of flame detection are improved. Smoke and fire detection program is developed on TMS320DM642 platform and video network transmission is achieved by i.MX27. The system has a good performance in different environments. It could well avoid disturbance of light and moving objects. The system has high real-time, quasi certainly and stability.
\end{abstract}

Keywords: TMS320DM642; i.MX27; Flame detection; Smoke detection

\section{Introduction}

Fire is a major problem with the development of human society, which has caused great loss to human society every year. There are many research results and products for fire detection. However, the general method of fire detection is based on smoke sensor, temperature sensor and light sensor to detect the flame and smoke [1]. But the general sensor used in large space building can only give the alarm when the fire occurs to a certain extent. That is to say, it could not achieve the early detection and alarm of fire [2,3]. With the popularity of video surveillance equipment and the extensive application of image processing, detection of flame and smoke detection is gradually being transited by the image processing of sensing devices. The intelligent video surveillance detection system can help people timely detection of a fire and greatly reduces the cost of manpower and material resources, thus, realizes efficient and accurate fire detection [4].

At present, a variety of research on video fire detection technology has been carried out at home and abroad. Infrared cameras or ordinary cameras are usually used in video flame detection. Infrared camera can monitor the infrared radiation of flame combustion, recognition rate is better, but because of its more sensitive to similar colors, will cause a certain false alarm rate. At the same time, flame and smoke detection based on ordinary camera is developing slowly due to its low cost. However, due to the limitations of existing algorithms, flame detection can't achieve very stable results. At present, the main algorithms of flame recognition based on image 
processing include flame feature extraction, circularity analysis, sharp angle feature analysis, area change and so on in the visible image mode [5]. Like reference 6 designs a fire video monitoring system based on ARM \& $\mu \mathrm{C} / \mathrm{OS}-\mathrm{II}$, and uses smoke sensor to detect fire [6]. There are some systems based on the DSP program, the literature 7 using DSP to achieve video signal acquisition, processing and transmission [7], there are also FPGA+DSP programs [8]. Smoke detection algorithm also has many achievements, such as the classification coding detection technology proposed by Nobuyuki [9], as well as the optical flow velocity field detection algorithms proposed by Kopilovic [10]. Many domestic studies are based on forest fire smoke. Because of the large spread of smoke caused by forest fires, it is not suitable for the smoke detection in the early stage of indoor fire.

The system uses TMS320DM642 of TI and i.MX27 of Freescale, through the division of labor between the DSP and arm, realizes video capture, smoke and fire detection processing, compression and network transmission. In the algorithm, the system uses the background subtraction algorithm based on OTSU threshold segmentation and smoke color, energy measurement and structural similarity metric algorithm for smoke detection. Flame detection uses dual band of infrared and visible light, which overcomes the disadvantages of using an infrared camera alone or a conventional visual camera for detection, reducing the false alarm rate.

\section{The Building of Hardware Platform}

DM642 is a high performance digital media processor, the CPU architecture VelociT1.2 is with an upgraded version of the advanced very long instruction word (VLIW) TMS320c64x DSP core VelociT, eight 32-bit instructions per cycle operation, and has rich peripheral interfaces. I.MX27 is a low-power, high performance Multimedia application chip made by Freescale, and is widely used in intelligent transportation systems, video surveillance, video telephony and other industries. Its kernel is ARM926EJ-S, with main frequency up to $400 \mathrm{MHz}$. It has a hardware video codec module, while integrated fast Ethernet control module (FEC), just outside the Ethernet PHY chip to achieve Ethernet communications, support $10 / 100 \mathrm{M}$ adaptive Ethernet interface.

System hardware block diagram as shown in Figure 2.1. The system capture twoway video signal of the visible light and the infrared light through decoder via DM642 video port. Video signals are stored in the SDRAM Video signal through the DMA mode, so that they could be used by the next program. DSP runs smoke detection algorithm for video signal processing, to determine whether there is smoke. The processed image is output to the ARM module which is running Linux operating system through the DSP video port. Using H.264 coding module embedded in i.MX27 to complete the video compression and then transmitting to the host computer monitoring program through network. 


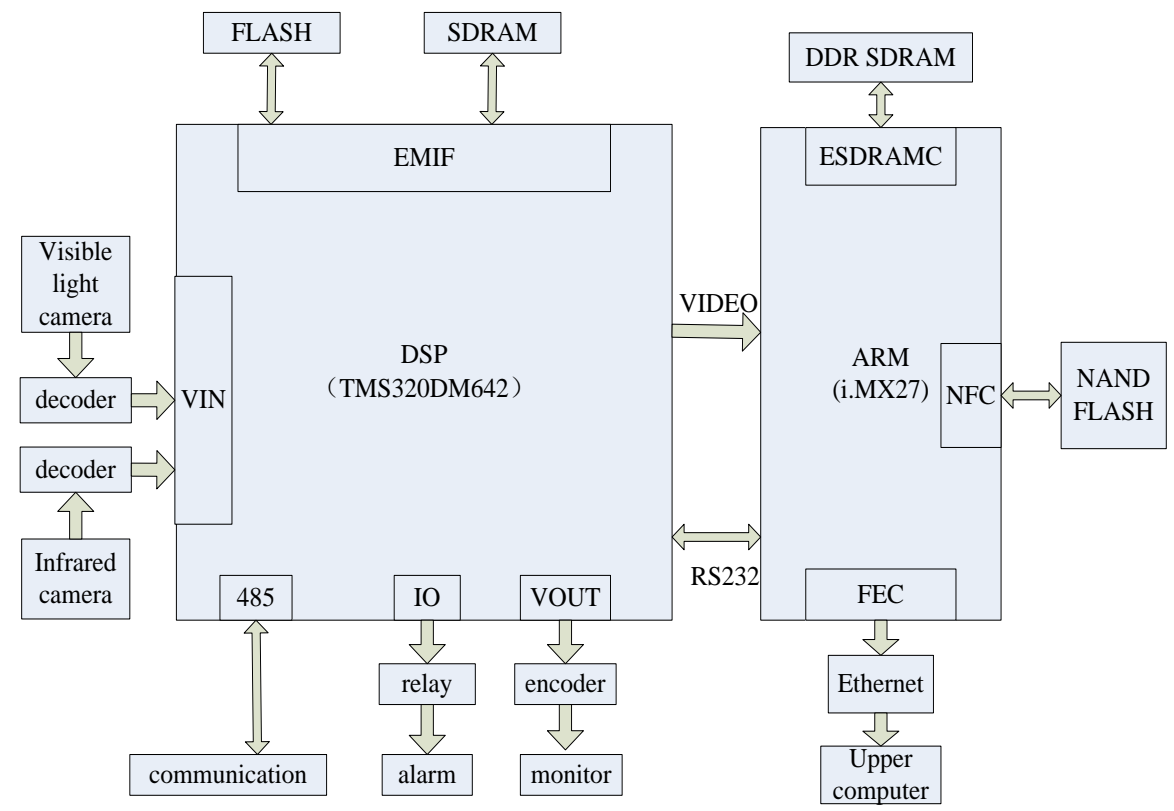

Figure 2.1. System Hardware Overall Block Diagram

\section{System Software Architecture}

DSP using complex instruction set is responsible for video capture and processing, the ARM926EJ-S core using the reduced instruction set and running Linux operating system is responsible for H.264 video coding compression and network transmission. The system board integrates ARM and DSP in one board, which has great advantage in stability.

The software architecture of the system is shown in Figure 3.1, which mainly includes serial port configuration program, video smoke detection processing algorithm program and alarm processing program. I.MX27 based on the Linux operating system is used to develop H.264 hardware compression program and the use of RTP protocol video network transmission program.

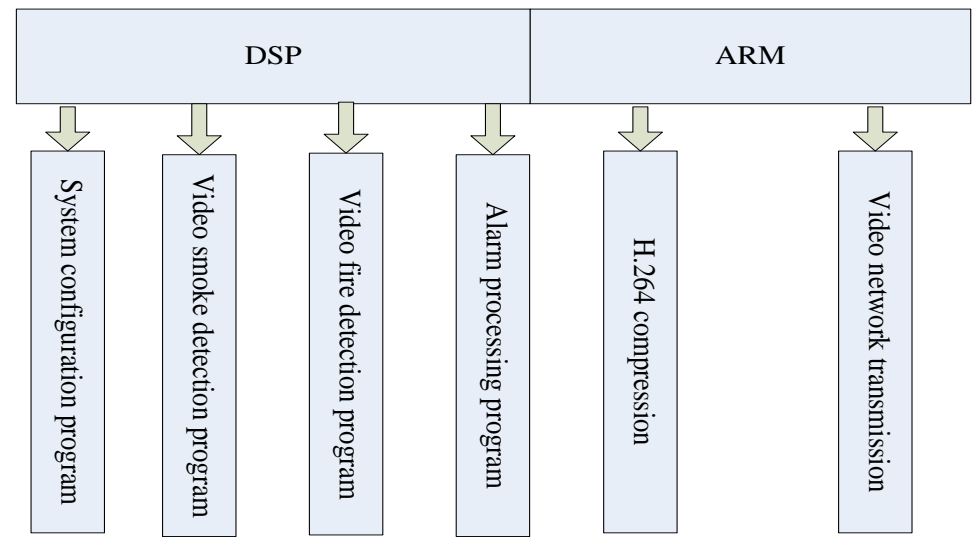

Figure 3.1. System Software Architecture Diagram 


\section{Smoke Detection Algorithm}

Smoke detection algorithm is the core part of the system. System collects video streaming through the video decoder chip. The video stream which is processed by the video decoder chip is stored in SDRAM by EDMA mode. Video information is stored in YUV422 format. The OTSU automatic threshold segmentation algorithm is used to process each frame image in the video stream and find suspicious motion image block. Then using the color measurement algorithm and the energy measurement algorithm based on wavelet transform to detect whether the suspicious primitives are the smoke elements. If the smoke element is found, the marker box is used to identify it and the alarm signal is given. Taking into account the real-time and accuracy of the system after several tests, the final choice of the size of the image block is $17 * 17$ after many tests. The following discussion is based on the $17 * 17$ image block. The flow chart of smoke detection algorithm is shown in Figure 4.1.

\subsection{Smoke Suspicious Area Extraction}

Camera position of the system is stationary. To detect suspicious smoke area, we need to build up the background model for the continuous video frame sequence. And then performs a differential operation on the background image and the current frame image. The selected threshold will be used to identify the area of movement. The background difference formula is:

Moving_pixels $(x, y)=\left\{\begin{array}{cl}255 & \text { if }|C P(x, y)-B P(x, y)|>T h \\ 0 & \text { else }\end{array}\right.$

Where $C P$ is the current video frame, $B P$ is the current background image, $T h$ is the OTSU threshold of the difference image, Moving_pixels $(x, y)$ indicates the difference detection results, this paper uses the pixel value of 255 to represent the moving target pixel point, 0 represents the background pixel point.

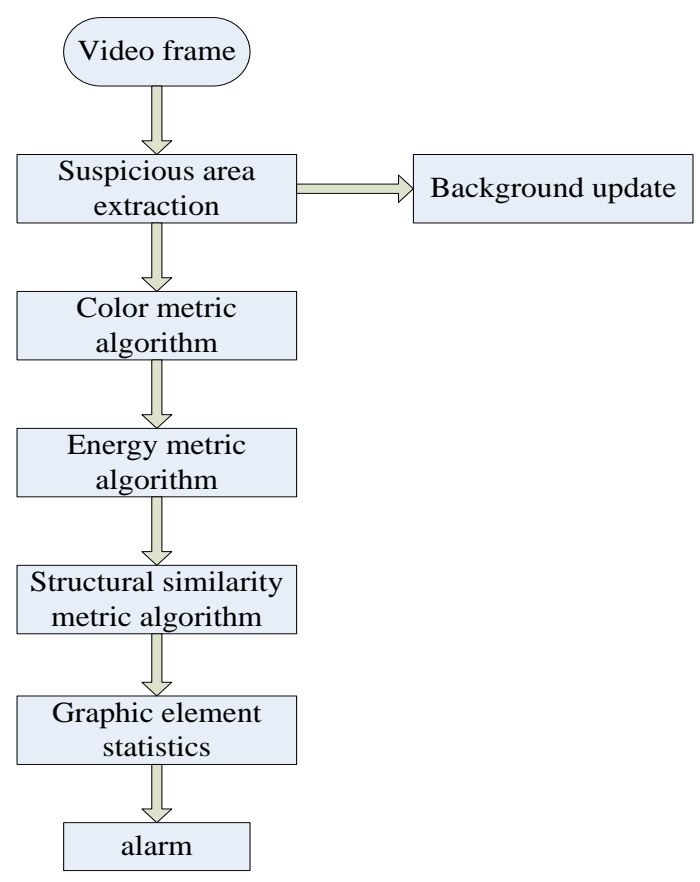

Figure 4.1. Flow Chart of Smoke Detection Algorithm 
Threshold selection is very important. This system uses the OTSU automatic threshold segmentation method. The method is simple and efficient. It could select a good threshold in the absence of a priori knowledge. The specific steps of OTSU are as follows: (1) From the gray-scale histogram of the difference image, we get the mean value of the difference image and the probability distribution of the gray level. (2) Traverse the gray level on each of the gray value "k". (3) Select value "k" as a threshold value, and the image pixel points are divided into foreground and background. And calculate the proportion and mean of the foreground and background points, and then calculate the interclass variance when the value is the threshold value. (4) According to the results, the corresponding gray level is selected as the optimal threshold when the variance between classes is taken as the maximum. Note that OTSU uses the value of inter-class variance to represent the difference of image gray level [11]. Taking into account the presence of noise disturbance in the video frames and the moving target in the presence of close to the background color, these factors may make the result of moving target detection is not very well. Therefore, it is necessary to carry on the post processing, which can be smooth filtering and morphological processing. The system selects expansion and corrosion algorithm. They can make the image more smooth, eliminate small targets and separate narrow connected objects.

\subsection{Smoke Color Measurement Algorithm}

In image analysis, the color model is very important. Commonly used color models are RGB model, HSI model and YUV model. The RGB model represents three colors: red, green and blue. Three channel color range is $[0,255]$. The HSI model uses hue H, saturation S and intensity I to describe color features. YUV model separates luminance and chrominance signals. Because of the sensitivity of the human eye to the brightness change is greater than the sensitivity of the color change, so the brightness signal can be used in image processing to do a lot of color analysis pretreatment. Analysis of the initial fire smoke, it can be found that the initial temperature is low, the material is not fully burning, the smoke color is thin, usually gray white. The video decoder of the system is TVP5150AM1. It outputs 8-bit ITU-R BT.656 data and separates a YUV signal components via a DM642 video port. Therefore, considering the system's realtime requirement, the YUV color model is selected and its format is YUV422, which includes luminance signal $\mathrm{Y}$ and chrominance signals $\mathrm{U}$ and V. Certainly, in order to improve the effectiveness of the system, we can also use other color models, but the DSP floating-point operation will weaken the real-time requirements of the system.

Before and after smoke, the intensity of $\mathrm{Y}$ component and saturation $\mathrm{S}$ component (HIS color model) of the data distribution has obvious change in trend, and the distribution is more concentrated. Taking into account the requirements of the real-time process, the system only uses the $\mathrm{Y}$ component, taking the ratio of the $\mathrm{Y}$ component values of the current suspicious image block and the $\mathrm{Y}$ component values of the corresponding area in the background image as a measure value. The formula for calculating the energy of suspicious image block is (2). If the ratio is less than the set threshold value, it passes through the color criterion and the current suspicious area information will be saved to the buffer.

$E(Y)=\sum_{x} \sum_{y} Y^{2}(x, y) \quad$ where Moving_pixels $(x, y)=255$

\subsection{Smoke Energy Measurement Algorithm}

In order to try to avoid false detection, the energy of smoke suspicious elements which has passed through the color criterion will be detected. In the early period of fire, smoke appear translucent, content of image high frequency will attenuate [12]. 
Through the time domain and frequency domain, it is clear to see the attenuation of high frequency energy in the image under the influence of smoke. Also it could be regarded as the judgment of smoke.

Wavelet transform has a wide range of applications in the field of image processing. The system uses one-dimensional wavelet transform to decompose image, extracting high frequency part of the image [13]. The definition of high frequency energy is the sum of vertical, horizontal and diagonal power spectrum of the wavelet decomposition. The formula is (3).

$$
H W=\sum_{i=H, V, D} \sum_{m} \sum_{n}\left|W_{\varphi}^{i}(J-1, m, n)\right|
$$

The ratio of high frequency energy of the foreground and background elements obtained from wavelet transform is defined as the energy analysis. Comparing the ratio and the threshold value of the program, the image area is identified as the smoke element and is placed in the smoke element buffer only if the ratio passes through energy measurement algorithm. In this paper, the wavelet model for the analysis of smoke characteristics of energy for the following considerations: (1) two-dimensional image of the fast wavelet transform time complexity is low, more efficient, which is more essential for DSP. (2) The wavelet transform overcomes the shortcomings of single-resolution Fourier transform.

\subsection{Smoke Structural Similarity Metric Algorithm}

Structural similarity index (SSIM) is proposed as an objective evaluation method of image quality, which adopts the whole reference contrast of the original image and the defective image without distortion. According to human visual perception, the structured information in the scene is extracted, and the quality assessment method of image structure information attenuation is introduced. The metric value of SSIM is (4):

$\operatorname{SSIM}(x, y)=\frac{\left(2 \mu_{x} \mu_{y}+C_{1}\right)\left(2 \sigma_{x y}+C_{2}\right)}{\left(\mu_{x}^{2}+\mu_{y}^{2}+C_{1}\right)\left(\sigma_{x}^{2}+\sigma_{y}^{2}+C_{2}\right)}$

Where $\mu_{x}, \mu_{y}$ is the brightness mean value. $\sigma_{x}, \sigma_{y}$ is the covariance of brightness. $C_{I}$ and $C_{2}$ are constants.

In the evaluation of image quality, the local area is usually calculated by SSIM instead of the global metric. When the mean value of the structural similarity between the suspicious and historical elements is larger than the threshold value, it is considered that the suspicious element has passed the SSIM's smoke discrimination analysis.

\subsection{Background Modeling}

Smoke algorithm needs to build a background for the use of differential images. At the beginning of the program, we take a picture as the background at the right time, such as $100^{\text {th }}$ frame. However, the background is uncertain, so we need to update the background in real time, so that the system can adapt to the impact of light and other interference on smoke detection. The weighted average of each pixel in the current frame and background frame is used to realize adaptive background updating. Since the current frame contains motion foreground, so we can avoid updating the moving part into the background in the future, we only need to update the pixels in the current frame. The formula for the background update is shown in (5).

$$
C B(x, y)=\left\{\begin{array}{cl}
\alpha C P(x, y)+(1-\alpha) B P(x, y) & \text { if Moving_pixels }(x, y)=0 \\
B(x, y) & \text { otherwise }
\end{array}\right.
$$


$\alpha$ is the update factor, the larger the background update the faster. But too much value will affect the smoke judgments, so the need for appropriate value, the general value of $0.001-0.01$.

\subsection{Error Detection Analysis}

Since the smoke of the early development of the fire is gray and translucent, so the smoke detection algorithm is more susceptible to interference, such as background color, transparent glass, indoor lighting changes, etc. Procedures are very sensitive, so it is easy to cause error detection. Therefore, it is necessary to improve the performance of the algorithm according to the appropriate threshold of the environment. After many experiments, the adaptive threshold segmentation algorithm based on OTSU and the smoke color and energy measurement algorithm can avoid the error detection in most cases.

\section{Fire Detection Algorithm}

In many domestic and foreign studies, many image based flame detection is based on visible light analysis. In this paper, the flame detection system based on dual band has better robustness than the general flame recognition system based on image processing. Firstly, we can get infrared distributed information from the infrared camera, and determine the high-temperature region and its suspected degree of flame. Secondly, we can get color information from the visible information of ordinary camera in the high temperature zone corresponding suspected flame of infrared picture. Then judge the current high temperature area is containing the flame or not. Using infrared camera to determine the location of the high temperature region, we need to extract high brightness infrared pixel from each picture respectively, scan contour, calculate sharp and get other information to determine the cumulative data for each suspected flame region. Collecting the visible information of the ordinary camera, obtain binarization information and contour scanning of the visible light color of the corresponding region of the flame region in the infrared processing, access to the area of information, and compare with the infrared corresponding suspected flame area, to verify whether it is a true flame. If there is flame area, it will be marked out and displayed. Algorithm flow chart is shown in Figure 5.1.

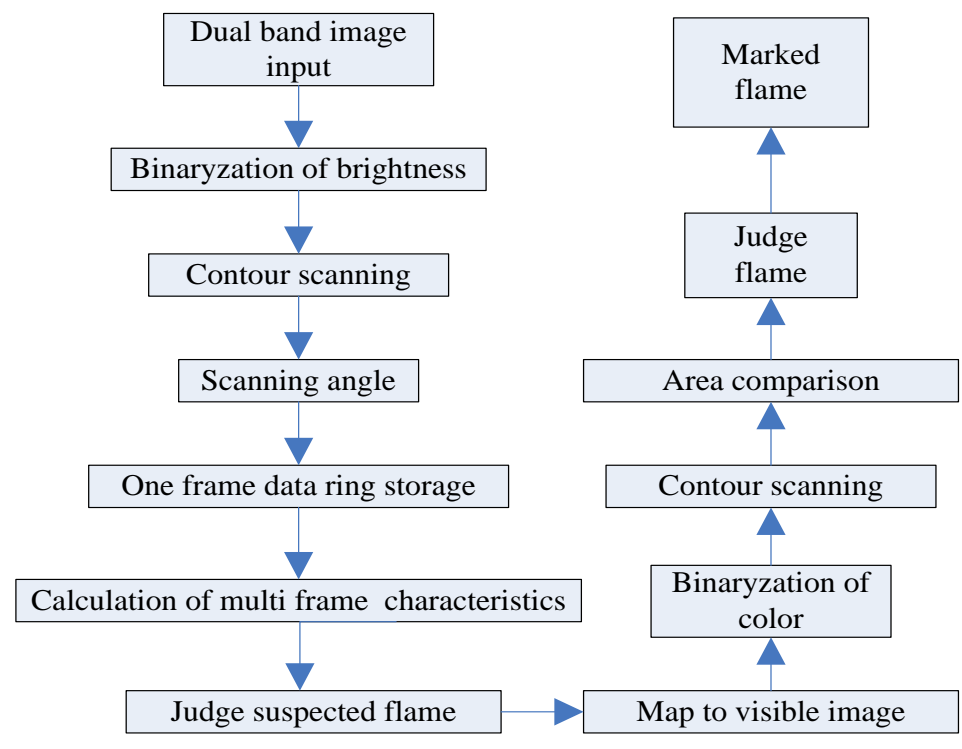

Figure 5.1. Software Framework of Fire Detection 


\subsection{Binarization Algorithm}

For the binarization algorithm, the two cameras are different. The ordinary camera is the visible light image information, the system is the collection of visible light information, which is based on the color criterion. But the infrared camera collects infrared image information, and therefore to judge the high temperature region, it only needs to make judgments of the brightness threshold, and the binarization algorithm is to choose high brightness pixels.

For example, suspicious pixels of the ordinary camera meet the formula (6)-(8), where $Y(x, y), C b(x, y)$ and $C r(x, y)$ is the brightness, the blue color difference value and the red color difference value of the points in the position $(\mathrm{x}, \mathrm{y})$, respectively. $\tau$ is a constant.

$$
\begin{aligned}
& Y(x, y)>C b(x, y) \\
& C r(x, y)>C b(x, y) \\
& F_{\tau}(x, y)=\left\{\begin{array}{lll}
1, & \text { if } & |C b(x, y)-C r(x, y)| \geq \tau \\
0, & \text { otherwise }
\end{array}\right.
\end{aligned}
$$

\subsection{Contour Scanning Algorithm}

The image obtained from the infrared camera and the common camera needs to be scanned by the contour algorithm to determine the flame boundary contour.

In the two valued image, there are a lot of suspected flame pixel points (hereinafter referred to as the image points) constituting the connected domain (hereinafter referred to as the suspected flame area). Contour scanning algorithm scans area of images needed to be scanned according to shun clockwise, getting all boundary points of each suspected flame region in the memory, and storing it in the cache. Flame detection is based on this. At the same time, the maximum and minimum values of horizontal coordinate and vertical coordinate of all points of the suspected flame area are recorded (that is, the upper left corner and the lower right corner of the suspected area). The boundary length of the suspected flame area (i.e., the area perimeter) is recorded, too.

As is shown in Figure 5.2, the upper left coordinate ( $\mathrm{x} 0, \mathrm{y} 0)$, the lower right corner $(\mathrm{x} 1, \mathrm{y} 1)$, the two points determine the location of the suspected flame area, marking a rectangular frame of the approximate area of the suspected flame area.

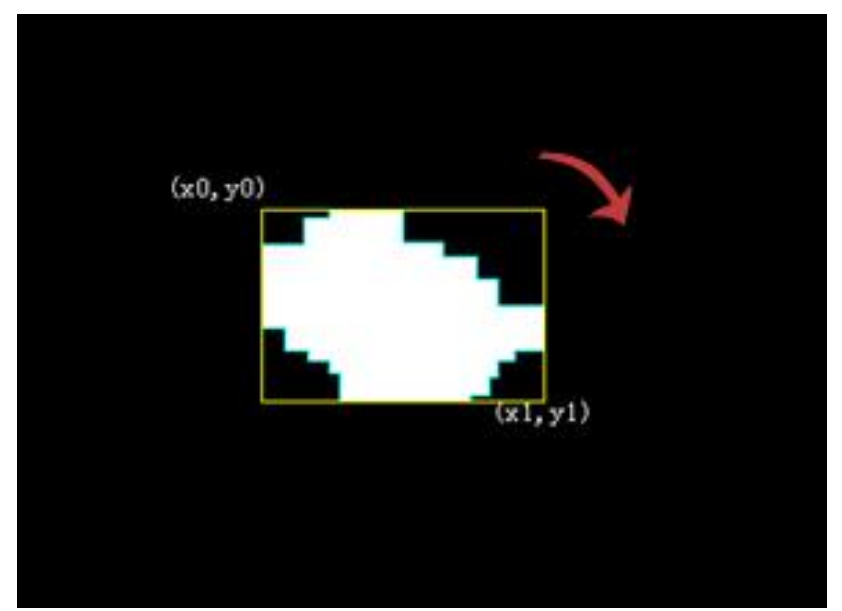

Figure 5.2. Contour Scanning Algorithm Framework 


\subsection{Suspected Flame Area Identification Algorithm}

Suspected flame area identification algorithm is based on Contour scanning. Through Contour scanning, we have get sharp quantity, area, perimeter and other information of the suspected flame area. Through a large number of experiments, we found that in the continuous video frames, there is a certain change of laws of sharp quantity, area and perimeter of flame area, which is different from other suspicious areas and can be used as a basis for judging the flame. Among them, the following research angle algorithm interface method: Firstly, six boundary points are taken from the left and right directions of the current boundary point to form the current judgment area. Secondly, preliminary judgment is made that the height of the 12 boundary points in the left-right direction is at least lower than the current boundary point. At the same time in the left and right sides in accordance with the three boundary points of the interval to take two sampling points. If the preliminary judgment condition is satisfied, proceed to the next step. As shown in Figure 5.3: If the height of A1 and A2 are lower than the height of $\mathrm{A}$ and the height of $\mathrm{A} 3$ is lower than the height of $\mathrm{A} 1$, and the height of A4 is lower than the height of A2, the current judgment area is approximately triangular. Finally, if the height and width of the approximate triangles are sufficient, the current judgment region is considered to be a sharp corner. Here the approximate height and width can be used to judge, the formula is shown in formula (9) and (10).

$H=\sqrt{\left(\frac{x_{3}+x_{4}}{2}\right)^{2}+\left(\frac{y_{3}+y_{4}}{2}\right)^{2}}$

$W=\sqrt{\left(x-\frac{x_{3}+x_{4}}{2}\right)^{2}+\left(y-\frac{y_{3}+y_{4}}{2}\right)^{2}}$

Where $x 3$ and $y 3$ are the abscissa and ordinate of point A3, x4 and $y 4$ are the abscissa and ordinate of point A4 respectively, and $x$ and $y$ are the abscissa and ordinate of point A respectively.

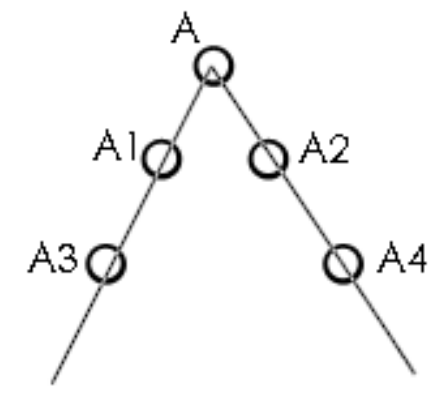

\section{Figure 5.3. Angle Algorithm Sampling Points}

According to the characteristics of contrast flame and non flame, the following conclusions are drawn: (1): Flame area, height standard deviation and perimeter has obvious difference between fire and non fire, relatively, flame area standard deviation is large. (2): The circular degree can distinguish the general flame and stable flame. The stable flame like candles, the circular degree is higher. (3): As for the mean and standard deviation of the number of sharp corners, the mean value of the flame is much larger than that of the non-flame and the standard deviation is larger too. However, due to the flame shape with randomness, it is inevitable that the sharp angle mean value is too small. For example, the candle flame tip angle is generally 1 , the standard deviation 
is generally 0. In Reference [14], a sharp angle characteristic of a flame is proposed. The suspected flame area identification algorithm of this system is based on these.

\section{System Debugging}

Smoke algorithm was tested in different environments. In different test contexts, we identify moving objects and smoke. Environmental changes include indoor and outdoor lighting changes, background color changes.

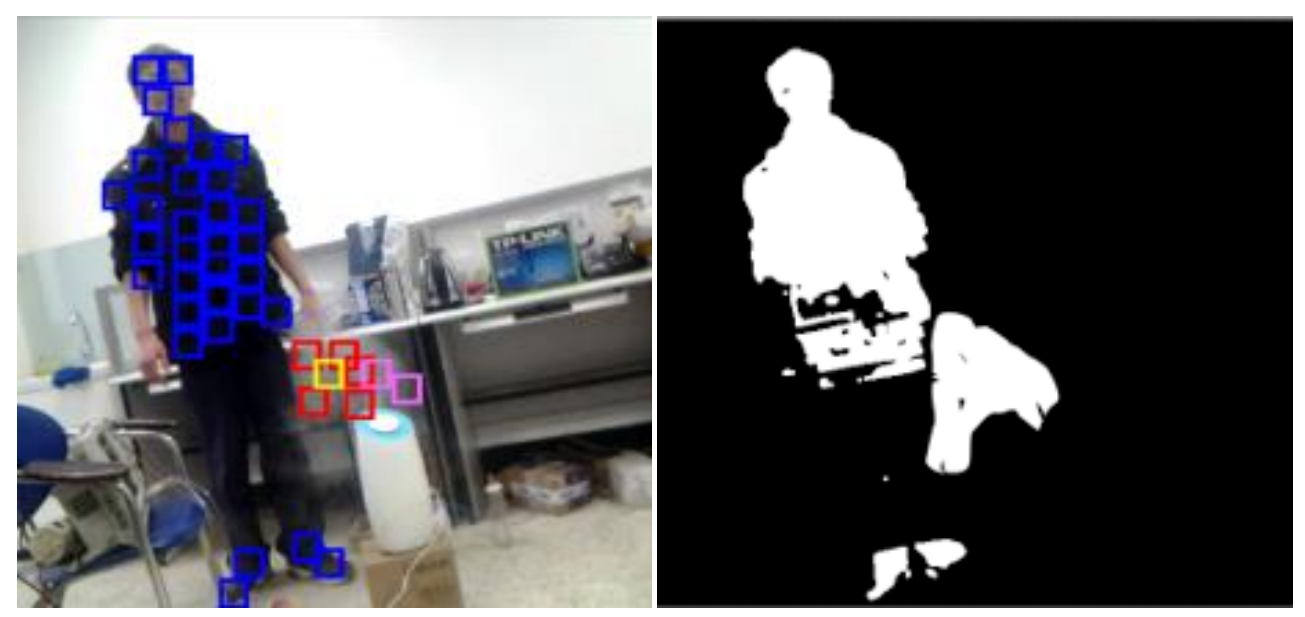

Figure 6.1. Foreground Image and Motion Detection Image

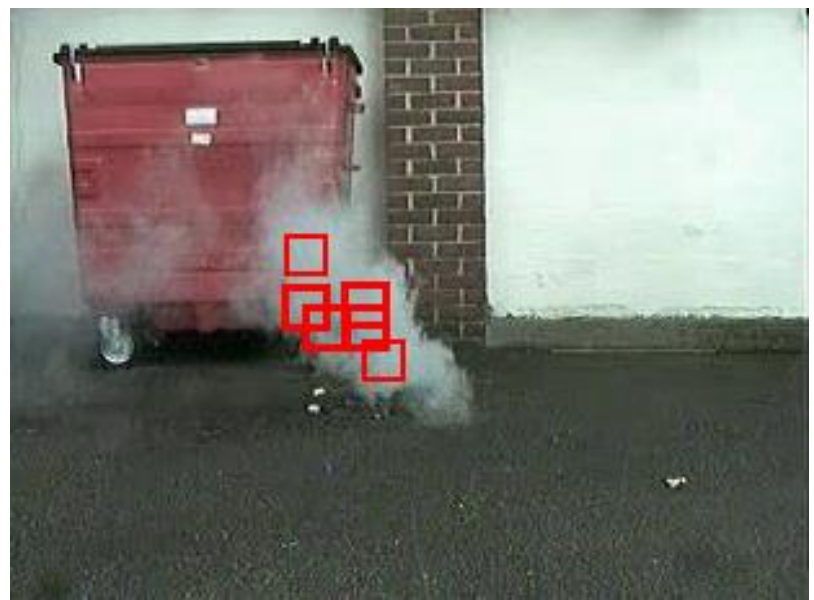

Figure 6.2. Effect of Outdoor Smoke Detection 


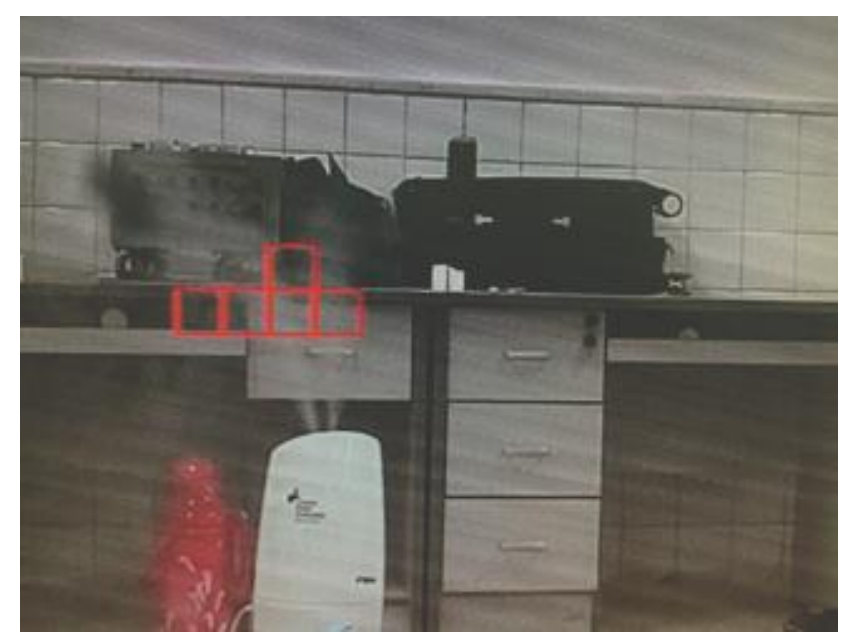

Figure 6.3. Effect of Smoke Detection in Low Light

Figure 6.1 is a screenshot of the actual operation of the system, smoke is simulated with the humidifier. Image block labeled by blue box represents that it only passes through the detection of moving objects. Image block labeled by yellow box represents that it passes through the detection of motion area detection, smoke color measurement and smoke energy measurement algorithm. Image block labeled by pink box represents that it passes through the detection of motion area detection, smoke color measurement and smoke energy measurement algorithm. Image block labeled by red box represents smoke area that is detected.

Figure 6.2 is a screenshot of the actual operation of the system where smoke is occurred outdoor. The background environment in Figure 6.3 is relatively white, and the smoke effect from the humidifier simulation is good.

Figure 6.4, 6.5 is an infrared image and the corresponding binarization image of the flame in an experiment. Figure 6.6 is the screenshot of the flame detection results. It can be seen from the figure that flame can be effectively detected.

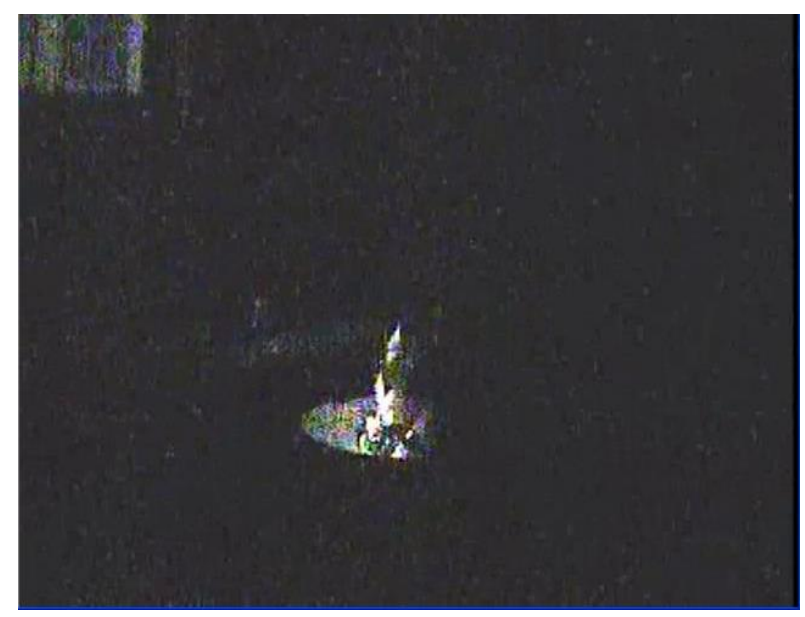

Figure 6.4. Field Image (IR) 


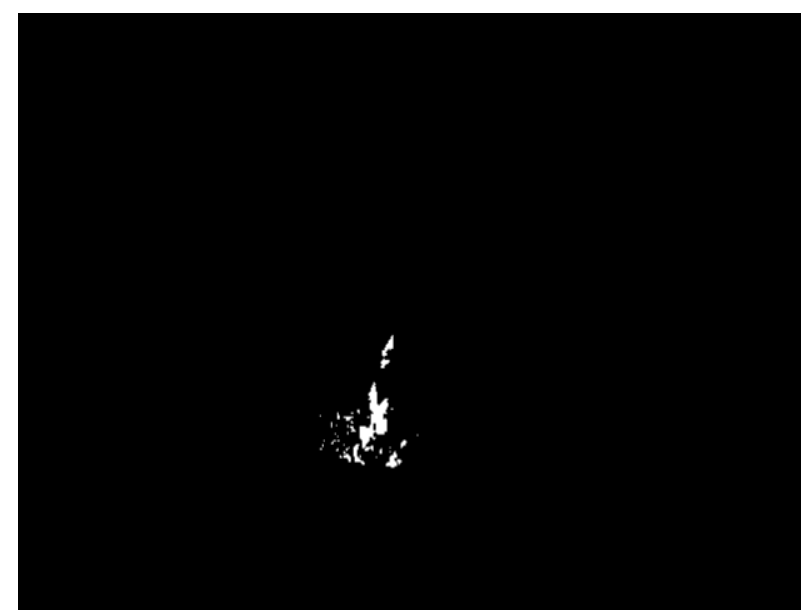

Figure 6.5. Binarization Image

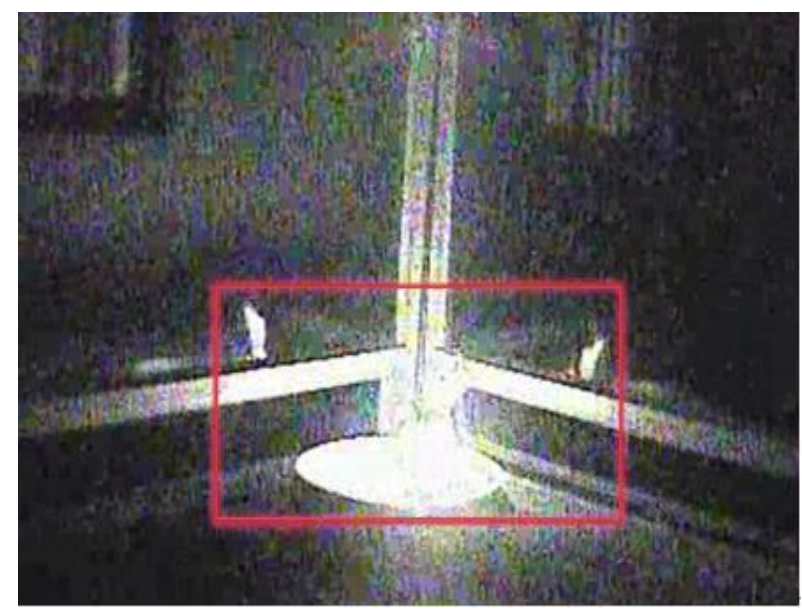

Figure 6.6. Flame Area Identification Image

\section{Conclusion}

A network video smoke and fire detector based on DM642 and i.MX27 is designed and implemented. The debugging of smoke and fire detection algorithm is completed. The smoke recognition algorithm designed in this paper can adapt to the change of the environment, and solve the problem of the traditional smoke recognition. In the field of flame detection, the dual band design also improves the accuracy and stability of traditional flame recognition based on image processing. The system is small in size, low in power consumption, and its service life is greatly improved. The system is mainly for large space, such as weapons and ammunition warehouse, shopping malls and other places which needs to strictly control the fire and make contributions to intelligent security.

\section{Acknowledgments}

This research was supported by Science and Technology Program of Lianyungang, China (No. SH1441). 


\section{References}

[1] W. Tao, L. Yuan and X. Zhenping, "Flutter Analysis Based Video Smoke Detection", Journal of Electronics \& Information Technology, vol. 3, no. 5, (2011), pp. 1024-1029 (in Chinese).

[2] B. C. Ko and J. O. Park and J.-Y. Nam, "Spatiotemporal bag-of-features for early wildfire smoke detection", Image and Vision Computing, vol. 31, no. 10, (2013), pp. 786-795.

[3] H. Maruta, A. Nakamura and F. Kurokawa, "Smoke detection in open areas with texture analysis and support vector machines", IEEJ Trans Electron Eng, vol. 7, no. S1, (2012), pp. 59-70.

[4] Alejandro O. B., Leonardo M. G. and Gabriel S. P., "Improvement of a Video Smoke Detection Based on Accumulative Motion Orientation Model", Electronics, Robotics and Automotive Mechanics Conference. IEEE, 2011, (2011), pp. 126-130.

[5] Q. Gaolin, "Research on Image Flame Feature Selection and Recognition Algorithm in Complex Large Space", Xi'an University of Architecture and Technology, (2015) (in Chinese).

[6] L. WenJiang, T. Yong and L. Nan, "Fire Compound Detection System Based on ARM\& $\mu$ C/OS- II", Application of computer system, vol. 20, no. 3, (2011), pp. 234-237(in Chinese).

[7] X. Zhenping, W. Tao and L. Yuan, "A new algorithm for fast detection of flutter analysis of video smoke", microelectronics and computer, vol. 28, no. 10, (2011), pp. 209-214(in Chinese).

[8] H. Sun and X. Bi, "Design of forest fire detection system based on embedded processor platform", Computer Engineering and Design, vol. 31, no. 6, (2010), pp. 1184-1187.

[9] N. Fujiwara and K. Terada, "Extraction of a Smoke Region Using Fractal Cording", Intenational Symposium on Communication and Information Technology, vol. 2, (2004), pp. 659-662.

[10] I. Kopilovic, B. Vagvolgyi and T. Sziranyi, "Application of panoramic annular lens for motion analysis tasks: surveillance and smoke detection", Proceedings of 15th International Conference on Pattern Recognition, vol. 4, no. 3, (2000), pp. 714-717.

[11] S. Surit and W. Chatwiriya, "Forest Fire Smoke Detection in Video Based on Digital Image Processing Approach with Static and Dynamic Characteristic Analysis", Acis/jne International Conference on Computers, (2011), pp. 35-39.

[12] H. Maruta, Y. Kato, A. Nakamura and F. Kurokawa, "Smoke detection in open areas using its texture features and time series properties", IEEE International Symposium on Industrial Electronics, ISIE (2009), pp. 1904-1908(in Chinese).

[13] Huang K. and University C. J., "Overview of Image Processing Based on Wavelet Transform", Value Engineering, vol. 41, no. 2, (2015), pp. 69-75.

[14] C. Guoqing, J. Tianyue and F. Hanqing, "Fire Image Recognition Algorithm Based on Flame Angle", computer applications and software, vol. 12, (2013), pp. 52-55.
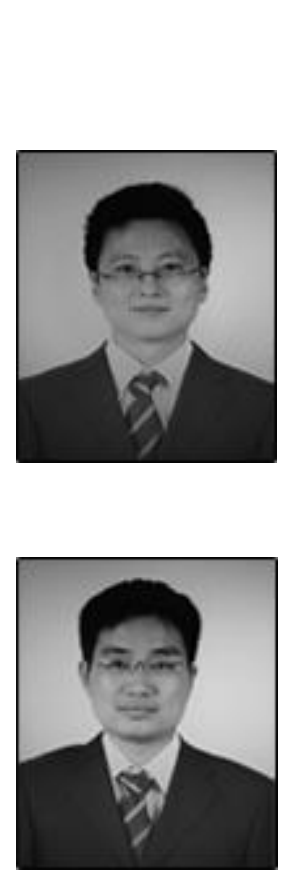

\section{Authors}

Hong Zhang, he is a senior engineer, and engaged in intelligent detection technology research. Graduated from Nanjing University of Science and Technology in July 2003, major in mechanical design and automation; Graduated from Nanjing University of Science and Technology in July 2005 with a master degree in mechanical design and automation; August 2005 to the China Shipbuilding Industry Corp No. 713 research institute.

Xianzhou Feng, he is a senior engineer, and engaged in intelligent detection technology research. Graduated from Nanjing University of Science and Technology in July 2003 with a bachelor degree in automation; In April 2006, graduated from the China Academy of naval ordnance, the theory and technology of weapon launch, master; May 2006 to the China Shipbuilding Industry Corp No. 713 research institute. 

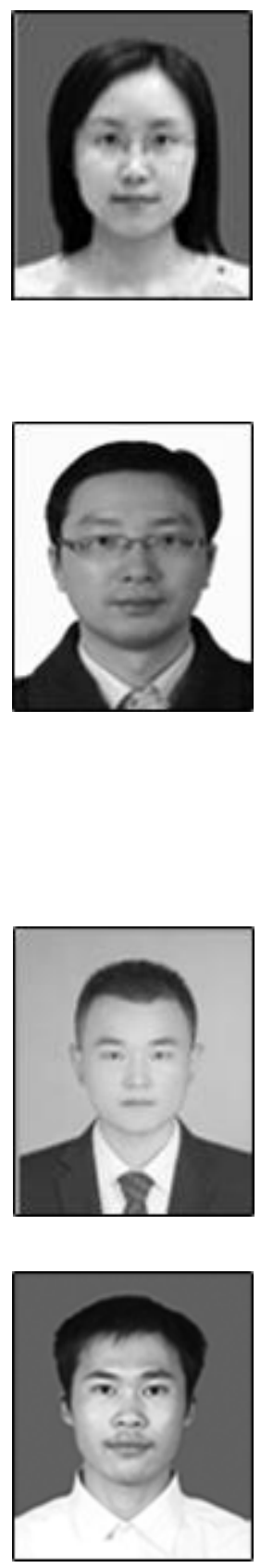

Ming Lyu, she received her BSc degree in Automatic Control in 2002, her MSc degree in Automatic Control in 2004 and her $\mathrm{PhD}$ degree in Control Theory and Control Engineering in 2007, all from Nanjing University of Science and Technology, Nanjing, China. She is currently a senior engineer in the Department of Simulation Equipment Business, North Information Control Institute Group Co., Ltd.. She current research interests include image processing, networked systems and stochastic control.

Jie Zhang, he received his BSc degree in Automatic Control in 2002, his MSc degree in Automatic Control in 2004 and his $\mathrm{PhD}$ degree in Control Theory and Control Engineering in 2010, all from Nanjing University of Science and Technology, Nanjing, China. From April 2013 to March 2014 he was an Academic Visitor in the Department of Information Systems and Computing, Brunel University, UK. He is currently an associate research fellow in the School of Automation, Nanjing University of Science and Technology. His current research interests include stochastic systems, networked systems, stochastic control and image processing.

Haiyan Li, Master's degree in reading at Nanjing University of Science and Technology, 2014.9-2017.4, Anhui Province, China. The main research direction is video image processing based on DSP.

Lei Liu, he is a Ph.D, candidate at the School of Automation, Nanjing University of Science and Technology. His research interests include image processing and networked control systems. 